\title{
Médecins et spécialistes
}

Le problème de l'unité de la médecine à Rome au $1^{\mathrm{er}}$ siècle ap. J.-C.

Par Philippe Mudry

La floraison des spécialités médicales à Rome sous l'Empire a fait l'objet d'un certain nombre d'études récentes ${ }^{1}$. Dans la liste fort longue qu'on en a dressée, certaines de ces spécialités apparaissent davantage comme des fantaisies caricaturales que comme l'expression d'une pratique médicale effective. Ainsi celle qui consiste, si on en croit le poète Martial ${ }^{2}$, à brûler les poils qui poussent de travers sur les paupières et irritent l'œil! G. Baader ${ }^{3}$ a remarqué à juste titre que les prétendus spécialistes que brocarde Martial dans son épigramme fameuse $(8,74)$ n'ont pas de désignation spécifique. En somme, des métiers sans nom. Cela doit au moins laisser planer quelque doute sur l'authenticité et le nombre réel de ces spécialités qu'une confrontation méthodique entre témoignages littéraires et témoignages épigraphiques réduirait vraisemblablement dans des proportions notables. Mais telle n'est pas notre intention ici. Sur le problème de la spécialisation médicale à Rome au début de l'Empire, qui est en fait celui de l'unité préservée ou perdue de la médecine, nous nous proposons d'examiner les témoignages de deux auteurs médicaux, Celse et Scribonius Largus. Ils présentent l'avantage, peu exploité jusqu'ici, d'offrir sur ce phénomène un regard de professionnel, puisque tous deux sont médecins ${ }^{4}$, en même temps qu'un témoignage contemporain et direct, puisqu'ils écrivent à Rome dans la $1^{\text {re }}$ moitié du $1^{\text {er }}$ siècle ap. J.-C.

Celse et Scribonius Largus affirment vigoureusement l'unité de la médecine en ses trois parties constitutives: diététique, pharmaceutique et chirurgie, division traditionnelle héritée de l'alexandrinisme. L'affirmation est identique chez l'un et l'autre auteur et formulée en des termes étonnamment proches: ces trois parties, disent-ils, sont si étroitement liées les unes aux autres qu'aucune d'entre elles ne peut être détachée de l'ensemble ${ }^{5}$.

On a souvent vu dans cette profession de foi l'expression d'une fidélité à un idéal hippocratique. Mais la formule est ambiguë. Le problème de l'unité de la médecine, qui ne peut se poser qu'à partir du moment où interviennent des divisions, est absent, à notre connaissance, des traités hippocratiques. Ces divisions sont l'œuvre de la réflexion alexandrine et de son goût des 
classifications, ainsi qu'en témoignent les nombreux schémas développés par les écoles médicales hellénistiques ${ }^{6}$. L'idéalisation d'Hippocrate, en qui les médecins alexandrins verront le père fondateur de leur art, modèle de savoir et de déontologie, a fait du maître de Cos, ici comme dans les autres domaines de la médecine, une référence nécessaire. En réalité, si la tripartition de la médecine en diététique, pharmaceutique et chirurgie est alexandrine, la revendication de son unité porte, nous semble-t-il, l'empreinte de Rome. Elle nous paraît être, dans le domaine de la médecine, l'expression de cet idéal encyclopédique de l'unité du savoir, hérité d'Alexandrie, certes, mais dont Cicéron fera à Rome le fondement de l'éducation. Selon le témoignage du De oratore $(3,132)$, dans lequel Cicéron regrette la fragmentation de la rhétorique, celle-ci doit être une et indivisible, tout comme les autres arts. Le propre des grands maîtres d'autrefois, affirme Cicéron, est d'avoir dominé chacun l'ensemble de sa discipline, qu'il s'agisse d'Hippocrate pour la médecine, d'Euclide et d'Archimède pour la géométrie, d'Aristoxène et de Damon pour la musique ou d'Aristophane et de Callimaque pour la critique littéraire. Toute spécialisation, qui est par définition renoncement à l'ensemble, est ressentie comme une atteinte à la discipline qui s'en trouve amoindrie et appauvrie ${ }^{7}$. C'est dans cette conception cicéronienne de l'unité du savoir, dont la fortune fut grande à Rome, que s'inscrit la revendication mainte fois répétée par Celse et Scribonius de l'unité organique de la médecine. Séparer les unes des autres les diverses parties de la médecine, soutient Scribonius, ne peut se faire sans porter préjudice à la profession tout entière ${ }^{8}$.

Il est révélateur, même si l'état fragmentaire dans lequel nous est parvenue la littérature médicale alexandrine doit forcément relativiser cette constatation, que les premiers auteurs médicaux à proclamer l'unité infrangible de leur art soient des Romains. Galien le fera aussi, mais plus d'un siècle après, et d'un point de vue différent dans lequel la composante doctrinale compte moins que le constat de l'extraordinaire dégradation scientifique et déontologique que la spécialisation excessive a, selon lui, entraînée dans la profession médicale.

Cela dit, deux questions se posent. En dehors de ces déclarations de principe, cette unité de la médecine se retrouve-t-elle pratiquement dans l'œuvre de Celse et de Scribonius? En d'autres termes, leurs traités révèlentils ou non une division au niveau des praticiens? Seconde question: dans quelle mesure ces traités sont-ils l'expression de la réalité médicale contemporaine? 
Rappelons tout d'abord combien, en l'absence de tout diplôme officiel, les contours de l'identité professionnelle du médecin sont flous. Si dans d'autres professions la sanction des faits, c'est-à-dire la bonne exécution de l'objet de l'art, est un critère suffisant à la reconnaissance publique de l'artisan - il est difficile, par exemple, de prétendre être potier ou forgeron quand on ne l'est pas, les faits se chargeant de dévoiler l'incompétence ou la supercherie - il n'en va pas de même pour la médecine, puisque la guérison du malade n'y dépend pas exclusivement de l'intervention du médecin. Celse avoue avec clairvoyance et modestie qu'en matière de maladie, on ne peut jamais vraiment savoir si la guérison est due au médecin, à la nature ou au hasard ${ }^{9}$. En outre, la guérison n'est pas toujours assurée, quelle que soit la valeur du praticien. Cette difficulté était d'ailleurs reconnue depuis longtemps par la réflexion sophistique sur les technai qui, dans sa bipartition des arts, avait rangé la médecine parmi les arts conjecturaux, ceux qui ne peuvent atteindre à coup sûr leur but ${ }^{10}$. En l'absence donc de critère concret ou formel établissant la qualité de médecin - on voit, à ce propos, avec quelle attention les sociétés de médecine veillent aujourd'hui sur le titre de médecin et quel soin jaloux elles mettent à dénoncer tout exercice illégal de la médecine, - l'affirmation de l'unité de la médecine telle qu'on la trouve chez Celse et chez Scribonius revêt également, nous semble-t-il, le caractère d'une revendication de l'identité propre de la profession médicale. Est médecin qui domine l'ensemble du savoir médical dans ses différentes parties. Qui, en revanche, ignore, délibérément ou non, un domaine de la médecine, peut certes s'intituler médecin, - comment, en effet, cela pourrait-il lui être interdit? - mais il doit être mis au nombre de ceux qui, comme les praticiens que condamne Scribonius parce qu'ils négligent l'usage des médicaments, ne sont plus médecins que de nom (nomine tantummodo medicorum) ${ }^{11}$. A plus forte raison, toute spécialisation se limitant à un organe, une affection, un type de traitement sera-t-elle exclue de la profession médicale. Il faut vraisemblablement voir là une des raisons du silence de Celse et de Scribonius sur ces spécialités que raillera un Martial et dont on peut imaginer que, à peine deux générations avant lui, certaines d'entre elles aient déjà existé. Il est vrai cependant que, à l'exception du médecin ocularius, anciennement attesté, et de deux inscriptions de l'époque de Claude concernant un médecin auricularius ${ }^{12}$, les témoignages de ces spécialités sont postérieurs à l'époque de Celse et de Scribonius.

Dans la préface qui introduit la partie de son traité consacrée à la chirurgie, Celse assure qu'un seul et même individu peut dominer l'ensemble 
de la médecine bien que, dans les faits, cela ne soit pas (ou plus) le cas. En effet, dit-il, étant donné que la division de la médecine est un fait, par quoi il faut entendre sa tripartition en diététique, pharmaceutique et chirurgie, le meilleur médecin est celui dont le savoir embrasse le plus de matière possible ${ }^{13}$. Doit-on en conclure que cette division de la médecine en trois domaines distincts a engendré également une division des praticiens? Chacune de ces trois parties de la médecine aurait-elle désormais ses propres représentants, en quelque sorte ses «spécialistes»? L'unité de la médecine ne serait plus dès lors qu'une vue théorique que rappellerait ici l'affirmation selon laquelle la valeur du médecin est proportionnelle à l'étendue de ses connaissances?

Une réponse peut nous être donnée par l'examen de la terminologie. Si la division de la médecine a donné naissance à une division identique des praticiens, ces derniers doivent avoir une désignation spécifique dérivée des domaines médicaux qu'ils pratiquent. Or que constate-t-on? Les livres diététiques et pharmaceutiques de Celse ne présentent pas d'appellation spécifique pour le praticien qui est désigné régulièrement par le terme général de medicus. Dans les livres chirurgicaux, en revanche, apparaît le terme de chirurgus, mais exclusivement dans la préface qui constitue pour ainsi dire la carte d'identité de la chirurgie et la présentation des raisons pour lesquelles cette dernière, comme la diététique et la pharmaceutique, est une partie autonome (non pas indépendante) de la médecine ${ }^{14}$. En dehors de la préface, chirurgus disparaît au profit de medicus, signe, nous semble-t-il, de la volonté de Celse de marquer, au-delà de sa spécificité, l'appartenance du chirurgien à la profession médicale. La terminologie de Celse révèle donc dans son œuvre non pas une tripartition des praticiens sur le modèle diététique - pharmaceutique - chirurgie, mais une bipartition qui correspond à la division ancienne, en tout cas nettement attestée chez Héraclide de Tarente ${ }^{15}$, entre affections internes et affections externes: la première catégorie relève essentiellement, mais pas exclusivement, de la diététique, la seconde de la chirurgie, tandis que la pharmaceutique se partage entre les deux types d'affections, ainsi que nous l'apprend un passage de Scribonius ${ }^{16}$ naguère peu clair, mais auquel la récente édition de $\mathrm{S}$. Sconocchia ${ }^{17}$, en le rétablissant dans son intégralité, a rendu toute sa signification. Faut-il dès lors parler, en recourant à des catégories modernes, d'une bipartition des praticiens entre généralistes et chirurgiens ${ }^{18}$, ce qui revient à donner au chirurgien le statut de spécialiste? La terminologie de Celse dans le couple medicus - chirurgus ainsi que son célèbre portrait du chirurgien (âge moyen, dextérité manuelle, acuité 
visuelle, force morale) ${ }^{19}$ peuvent inciter à le faire. Mais la terminologie de Scribonius nous amène à porter une appréciation quelque peu différente.

Si Celse, en effet, n'a pas de terme spécifique pour désigner le médecin qui traite les affection internes, les maladies, Scribonius, en revanche, qui distingue les mêmes deux types de praticiens dans l'exercice de la médecine, oppose au chirurgus le diaeteticus ${ }^{20}$. Il s'agit, il est vrai, d'un néologisme, puisque ce terme représente le premier emploi masculin substantivé de cette translittération du grec ${ }^{21}$. Mais le fait qu'il soit attesté chez Galien ${ }^{22}$ montre qu'il ne s'agit pas là d'une désignation spécifique inventée par Scribonius. Nous sommes même enclins à penser que son absence chez Celse peut être due à la réticence bien connue de l'auteur du De medicina à l'égard des termes techniques grecs. Il existe d'ailleurs une autre appellation pour désigner le médecin qui s'occupe des affections internes, elle aussi translittération du grec, mais postérieure à Celse et à Scribonius, celle de clinicus, attestée aussi bien littérairement qu'épigraphiquement ${ }^{23}$.

Chez Celse et Scribonius, l'ensemble de la profession médicale se divise donc en deux grandes catégories de praticiens, le diététicien et le chirurgien. L'apparition d'une désignation spécifique pour le médecin qui traite les maladies montre qu'il s'agit d'une catégorie de praticiens aussi nettement différenciée que celle du chirurgien. L'ampleur de leurs champs d'activité respectifs (affections internes, morbi - affections externes, ulcera uulneraque), le fait qu'elles ne limitent pas leur action à un organe, une affection précise non plus qu'à un type de traitement, puisque diététiciens et chirurgiens doivent également recourir aux ressources des autres parties de la médecine, rendent peu approprié l'emploi à leur sujet, comme on le fait d'ordinaire pour la chirurgie, du terme moderne de spécialité avec tout ce qu'il comporte de limitation et de restriction. $\mathrm{Ou}$, si on le fait, faut-il dès lors les distinguer nettement des innombrables spécialités qui limiteront leur intervention à un organe (p.ex. les yeux), une affection (p. ex. les fièvres) ou un traitement (p.ex. l'hydrothérapie)?

Celse et Scribonius ignorent la poussière de spécialités dont feront état les témoignages postérieurs, ainsi que nous l'avons signalé plus haut en indiquant les raisons possibles de cet état de fait. Il y a pourtant une exception: tous deux font état, à propos des affections oculaires, du medicus ocularius ${ }^{24}$, Scribonius utilisant même ce terme comme un substantif. Ainsi, quelle que soit leur position doctrinale sur l'unité de la médecine, ils admettent de facto l'existence d'une spécialité au sens strict du terme puisque limitée à un organe. En cela, ils ne font vraisembablement que s'incliner devant un fait. 
Les affections oculaires constituent dans cette première moitié du $1^{\mathrm{er}}$ siècle de notre ère un domaine de la médecine qui a acquis son autonomie et développé ses représentants propres ainsi qu'en témoignent Cicéron ${ }^{25}$ et des attestations épigraphiques qui remontent à l'époque d'Auguste ${ }^{26}$.

La médecine scientifique, nous voulons dire par là la médecine qui s'inscrit dans la tradition hippocratique et alexandrine, telle que l'incarnent à Rome Celse et Scribonius, reconnaît donc, même si c'est sur un plan différent, trois types de praticiens: le médecin diététicien, le médecin chirurgien, le médecin oculiste. Un siècle auparavant, le De oratore témoigne que cette tripartition de la pratique médicale a une existence effective. Cicéron y déplore, en effet, qu'il y ait désormais des médecins pour soigner les maladies, d'autres les blessures, d'autres encore les yeux ${ }^{27}$. Est-ce encore la situation qui prévaut à l'époque de Celse et de Scribonius ou l'état de la profession médicale que révèlent leurs œuvres n'est-il plus qu'une référence périmée au passé? Les facteurs d'éclatement semblent en tout cas s'amonceler dans une profession que menacent, selon Scribonius ${ }^{28}$, à la fois la négligence du public, qui se préoccupe davantage de frivolités que de la qualité du médecin auquel il confie sa santé, et la baisse de qualité de nombre de praticiens, ignorant la tradition et guidés par le goût du profit sans effort. Il est pourtant un témoignage qui nous montre que cette unité de la médecine que professent avec tant de conviction Celse et Scribonius pouvait encore se trouver concrètement réalisée dans un seul homme, ainsi que le prétend Celse ${ }^{29}$. Il s'agit de l'inscription funéraire d'un médecin de province, Merula d'Assise, qu'un certain nombre d'éléments nous permettent de dater selon toute vraisemblance du $1^{\text {er }}$ siècle ap. J.-C. ${ }^{30}$ et qui décrit en ces termes l'identité professionnelle du défunt dont elle perpétue le souvenir:

medicus clinicus, chirurgus, ocularius.

\section{Notes}

${ }^{1}$ M. Michler, Das Spezialisierungsproblem und die antike Chirurgie, Bern 1969. P. Platiel, Das Spezialistentum in der Medizin bis zum Ausgang der Antike, München 1977. G.Baader, Spezialärzte in der Spätantike. Medizinhistorisches Journal 2, 1967, p.231-238. K.-D. Fischer, Der ärztliche Stand im römischen Kaiserreich, Medizinhistorisches Journal 14, 1979, p. 163-166.

${ }^{2}$ Martial 10, 56, 4 .

${ }^{3}$ op. cit.p. 234. 
${ }^{4}$ La question de savoir si Celse a effectivement pratiqué la médecine importe peu ici. Son œuvre témoigne d'une connaissance vaste, précise et réfléchie de la tradition médicale antique. En ce sens, il est médecin.

${ }^{5}$ Celse, De medicina 5, préf. 2: omnes medicinae partes ita innexae sunt ut ex toto separari non possint.

Scribonius Largus, Compositiones 200: implicitas medicinae partes inter se et ita conexas esse ut nullo modo diduci sine totius professionis detrimento possint.

${ }^{6}$ Cf. K. Deichgräber, Die griechische Empirikerschule, Berlin 1930 (réimp. 1965), p. 288-291.

${ }^{7}$ Cicéron, De oratore 3, 132: distributione partium ac separatione magnitudines sunt artium deminutae.

${ }^{8}$ Cf. supra note 5 .

${ }^{9}$ De medicina 7 préf. 1.

10 Sur cette bipartition des technai, voir F. Heinimann, Eine vorplatonische Theorie der Techne, Museum Helveticum 18, 1961, p.123 sqq.

11 Compositiones préf. 9.

12 CIL VI, 8908 et 37752.

13 De medicina 7 préf. 5 .

14 ibid. 7 préf. $1-5$.

15 Héraclide de Tarente avait exposé l'ensemble de la thérapeutique en deux grands traités, l'un s'occupant des affections internes, l'autre des affections externes.

16 Compositiones 200.

17 Teubner, Leipzig 1983.

18 G. Baader, op. cit.p. 232: praktische Ärzte-Chirurgen.

19 De medicina 7 préf. 4 .

${ }^{20}$ Compositiones 200: ad diaeteticos... ad chirurgos.

21 Cf. Thesaurus linguae Latinae V, 948, 22.

${ }^{22}$ Galien, Thras. 24 (Script. min. III, p. 62).

23 Thesaurus linguae Latinae III, 1350, 12 sqq.

${ }^{24}$ De medicina $6,6,8 \mathrm{~A}$.

${ }^{25}$ De oratore $3,132$.

${ }^{26}$ Cf. H.Gummerus, Der ärztliche Stand im römischen Reich nach den Inschriften, Helsingfors 1932.

${ }^{27}$ Loc. cit.: fuisse tum alios medicos qui morbis, alios qui uulneribus, alios qui oculis mederentur.

28 Compositiones préf. 9-10.

${ }^{29}$ Cf. supra note 13.

${ }^{30}$ CIL XI, 5400 (= Dessau 7812). La référence au sévirat ainsi que l'ancien système de notation des nombres que présente cette inscription indiquent, pour sa datation, une fourchette entre les deux dernières décennies du $1^{\text {er }}$ siècle av. J.-C., époque où fut instauré à Assise le sévirat municipal, et les dernières décennies du $1^{\mathrm{er}}$ siècle ap. J.-C., au cours des. quelles fut définitivement abandonné l'ancien système de notation des multiples de mille. Nous devons ces précisions à notre collègue épigraphiste François Mottas que nous remercions très vivement. 


\section{Summary}

Celsus and Scribonius Largus positively affirm the unity of medicine considered in its three parts: dietetics, pharmaceutics and surgery. This claim is based on the encyclopedic and Ciceronian conception of the unity of learning. Their works however reveal a bipartition of practitioners (dieteticians-surgeons) which corresponds to the traditional division between internal and external affections. As for specialists in the narrow sense of the term (of an organ, an affection or a therapy) they know only the oculist. Thus they bear testimony to an actual tripartition of practitioners which Cicero one century before already noticed and deplored. Nevertheless some physicians may still join in their practice the three orientations and that is shown by the funeral inscription of Merula from Assisi (lst century A.D.).

Prof. Dr. Philippe Mudry

Faculté des lettres

Bâtiment central

CH-1015 Lausanne/Dorigny 